\title{
RESISTENCIA A LA FRACTURA RADICULAR ANTE FUERZAS COMPRESIVAS DE DIENTES TRATADOS ENDODÓNTICAMENTE Y RESTAURADOS CON TRES TÉCNICAS DE COMPLEMENTACIÓN
}

\author{
${ }^{1}$ Diana Inés Bernal Espinosa, ${ }^{1}$ Jorge Guerrero Home, ${ }^{1}$ Henry Galvis Galarza, \\ ${ }^{1}$ Martha Lucía Rodríguez Archila, ${ }^{1}$ Flor Victoria Sosa Peña, ${ }^{2}$ Antonio José Candela Gónzalez, ${ }^{3}$ Lilia Olaya Luengas. \\ ${ }^{1}$ Estudiante III Año Especialización en Rehabilitación Oral Universidad Santo Tomás, Bucaramanga, Colombia. \\ ${ }^{2}$ Especialista en Rehabilitación Oral U. Javeriana, Docente Universidad Santo Tomás, Bucaramanga, Colombia. \\ ${ }^{3}$ Ingeniera Facultad de Ingeniería Mecatrónica Universidad Santo Tomás, Bucaramanga, Colombia.
}

Autor responsable de correspondencia: Diana Inés Bernal Espinosa

Correo electrónico: dianabernale@hotmail.com

RESUMEN

Objetivo: Establecer si existe diferencias entre la resistencia a la fractura de dientes con postes de oro, núcleos en fibra de vidrio y complementador coronal.

Materiales y métodos: Se realizo un estudio experimental In vitro con una muestra de 45 dientes premolares que se dividieron en tres grupos iguales: núcleos colados en oro, postes en fibra de vidrio y complementador; se realizaron los tratamientos de endodoncia y los cortes coronales se embebieron en cubos de acrílico. Los dos primeros grupos se desobturaron dejando selle apical de $4 \mathrm{~mm}$. El núcleo y el poste se cementaron con cemento de resina dual. Al grupo complementador se reconstruyó coronalmente. Se prepararon en 360 grados y se cementarcon con cofias de metal base. Se aplicó la fuerza compresiva en una máquina universal de ensayos a velocidad de $0.05 \mathrm{~mm} / \mathrm{min}$, suspendida cuando se evidenció la caída de la curva traducida como falla.

Resultados: El 29\% del grupo de núcleo colado se fracturó a nivel medio y apical. Del grupo complementador se fracturó un premolar (7\%) a nivel medio. Se estableció dependencia estadísticamente significativa entre la técnica de complementación y la resistencia a la fractura y el nivel de fractura $(\mathrm{p}=0,041)$.

Conclusiones: Los dientes con núcleos colados resisten mayor fuerza pero presentan mayor probabilidad de fractura. Los dientes con postes en fibra resistieron menor fuerza aplicada y no se evidenció fractura. Los dientes con complementador presentaron menor resistencia a la fractura y un nivel de fractura no favorable. [Bernal DI, Guerrero J, Galvis H, Rodríguez ML, Sosa FV, Candela AJ, Olaya L. Resistencia a la fractura radicular ante fuerzas compresivas de dientes tratados endodònticamente y restaurados con tres técnicas de complementación. Ustasalud 2010; 9: 83 - 88]

Palabras claves: Postes, Modulo de elasticidad, Resistencia fractura.

\section{ROOT FRACTURE RESISTANCE TO COMPRESSIVE STRENGTH OF ENDODONTICALLY TREATED TEETH AND RESTORED WITH THREE COMPLEMENTARY TECHNIQUES}

\begin{abstract}
Objective: To establish a difference in the resistance to the fracture of teeth restored with casting gold post, glass fiber post and the system of coronal complementation.

Methods: An experimental In vitro study was done. Forty-five upper first premolars were selected and divided in three equal groups: gold type III post, glass fiber post and the system of coronal complementation. Each tooth was treated endodontically; coronal cuts were made and were put in acrylic resin cubes with a matrix of aluminum. The three groups were prepared coronally in 360 degrees and cement a metal cap base (remanium) calibrated 0.5 microns. Finally, subjected to compressive force on the Shimatzu universal machine with an initial load of 200 newtons at a constant speed of $0.05 \mathrm{~mm} / \mathrm{min}$ and the application of force at the time was retired graphically showed the collapse of the translated curve fails.

Results: Twenty nine percent of teeth with gold post presented fracture, half of this fracture was at medium level and the others were at coronal and apical level. In the group with the system of coronal complementation only one premolar presented fracture at the medium level (7\%). Statistical significant dependence was established between the technique of complementation and resistance to fracture and the fracture level $(\mathrm{p}<0,05)$.

Conclusions: Restored teeth with casting posts had better resistance to force but had a higher probability to fracture. Restored teeth with fiber glass post presented less resistance to force but did not present any fracture. Restored teeth with the system of coronal complementation presented less resistance to fracture and according to the level of fracture it was in a better level.
\end{abstract}

Key words: Post, Elastic modulus, Fracture resistance.

Recibido para publicación: 30 de noviembre de 2010. Aceptado para publicación: 20 de diciembre de 2010. 


\section{INTRODUCCIÓN}

En los últimos años se han realizado muchos estudios que han encontrado una mayor incidencia de fractura en los dientes con tratamiento de conductos y restaurados con núcleos colados, lo que convierte a las fracturas radiculares en un problema por la dificultad para ser diagnosticadas y para determinar su pronóstico. Devolver la resistencia al diente endodónticamente tratado es un gran desafío si se considera que el tejido dental es básicamente tejido conectivo bastante flexible y resilente. Cualquier material más rígido está atentando con los principios biológicos.

Hasta los años sesentas aún se creía que el tratamiento de conductos debilitaba a los dientes debido a la disminución en el contenido de humedad de su estructura y que le restaba un menor modulo de elasticidad, conduce a una mayor fragilidad mecánica frente a las fuerzas masticatorias. ${ }^{1}$ Linde (1995) encontró que un diente tratado endodónticamente, tiene apenas un $9 \%$ menos de humedad, con respecto a un diente vital, lo que no se considera clínicamente significativo. ${ }^{2}$

De igual manera, se ha demostrado que el abordaje endodóntico, más los procedimientos de instrumentación, le restan al diente un 5\% de rigidez en promedio, mientras que otros procedimientos invasivos son mas cuestionables en sus efectos sobre el tejido dentario. Se ha encontrado que una cavidad oclusal le resta un $20 \%$ de solidez al diente receptor; una cavidad mesio-oclusal o disto-oclusal un $46 \%$ y una cavidad mesio-ocluso-distal un 63\%.,4 En este sentido, parece que los procedimientos odontológicos en general que contemplan la pérdida de sustancia dentaria, debilita a los dientes; sin embargo un correcto tratamiento de conductos parece estar dentro de los procedimientos dentales menos deletéreos. $^{3}$

Otras complicaciones que se pueden presentar durante el proceso de la preparación radicular para recibir el núcleo son la contaminación bacteriana por exposición del medio oral durante el proceso de desobturación del conducto y el tiempo transcurrido para la cementación del poste por falta de un selle hermético del temporal y decementación del mismo, la perforación y creación de nuevas vías durante el proceso de desobturación, el debilitamiento y microfracturas generadas durante la preparación del conducto para recibir el poste y la pérdida del selle apical durante la desobturación para lograr la longitud del poste en vías de su retención. ${ }^{3,5}$

Además, se pueden presentar problemas debido a la falta de conocimiento de la anatomía radicular y la localización de las zonas de peligro por parte del rehabilitador al buscar un mayor diámetro del perno colado lo que aumenta el riesgo de fracturas de instrumentos dentro del canal e imposibilta el acceso para la elaboración del núcleo. ${ }^{6}$ Es importante considerar que en una eventual fractura radicular las posibilidades de recuperación dependerán en algunas situaciones del nivel de la fractura. Si esta es más coronal permitirá conservar una línea protésica a nivel del margen gingival o al ser subgingival podrá ser rehabilitado con una técnica de extrusión ortodóntica o cirugía de alargamiento coronal.,

El propósito de este estudio fue establecer la resistencia a la fractura ante fuerzas compresivas de dientes restaurados con tres técnicas de complementación diferentes.

\section{MATERIALES Y MÉTODOS}

Este es un tipo de estudio experimento de laboratorio de diseño In vitro, los dientes donados por fines ortodónticos fueron conservados en solución salina después de la exodoncia. Se seleccionaron 45 dientes primeros premolares maxilares que cumplían con los criterios de inclusión los cuales fueron verificados clínicamente y radiográficamente con la ayuda del microscopio Zeiss, Opm19 Schwenrkarm 3054, 4 aumentos).

Los dientes se limpiaron con scaler (Bobcat, Dentsplay de 30 vibraciones, 11 Svac. CE 0086, USA) con piedra pómez (Laboratorio León) cepillo de profilaxis y contrangulo (NSK) la muestra seleccionada se desinfectó en hipoclorito de sodio al 5.25\% por 5 minutos y lavados en agua destilada.

Los dientes fueron divididos al azar en tres grupos: 15 postes colados, 15 postes fibra de vidrio, 15 complementador coronal. La selección al azar se realizó con balotas de tres colores diferentes correspondientes a cada grupo de trabajo depositadas en una bolsa oscura y seleccionadas por una persona ajena a la investigación.

Un solo operador realizó los tratamientos endodónticos a todos los dientes con el fin de evitar sesgos de preparación. La apertura coronal se realizó con pieza de mano de alta velocidad (NSK Nakahishi. Inc., 700 Shumohinata, 322-8666 Japon) y fresa redonda de diamante número 4 (CNP. J 47.388.533 Brasil), se verificó la longitud de trabajo con lima 10 (Maillefer, Dentsply, Ballaigues, Suiza) a $1 \mathrm{~mm}$ del ápice radiográfico verificándose radiográficamente. La preparación del conducto se realizó con la técnica step-back, con irrigación abundante de solución de hipoclorito de sodio al 5,25\% y con una lima apical principal entre 25 a 30 (Maillefer, Dentsply, Ballaigues, Suiza). Los conductos se secaron con puntas de papel (Dent B.N.K. Bogotá, Colombia), se realizó 
la conometria (Maillefer, Batch 820, Ballaigues, Suiza) con verificación radiográfica (Radiología digital Trophy) y posterior obturación del conducto con cemento Top Seal (Maillefer, Ballaigues, Suiza) y con técnica combinada de condensación lateral y vertical con calor. Se verificó la obturación del conducto radicular dentro de los parámetros requeridos mediante el uso de radiología digital.

Se desobturaron con fresas Pesso (Mailleffer, Ballaigues, Suiza) números 3, 2 y 1, los dientes pertenecientes al grupo de postes colados, incluidas las $2 / 3$ partes de la raíz, dejando un remanente de gutapercha de $4 \mathrm{~mm}$ para lograr un selle apical de la endodoncia. Los dientes pertenecientes al grupo de postes prefabricados en fibra de vidrio (x-post Dentsply) se desobturaron y el conducto se conformo con la fresas Easy Post, Dentsply $\mathrm{CH}$ 1338 \# 1. Los dientes incluidos en el grupo de técnica de complementación no fueron desobturados.

Un solo operador realizó tres cortes a nivel coronal, un corte horizontal que eliminó el $50 \%$ de la corona, medido desde la línea amelocementaria hasta el vértice de la cúspide vestibular, un corte vertical desde el centro corona hasta $2 \mathrm{~mm}$ por encima del límite amelocementario y un corte horizontal $2 \mathrm{~mm}$ por encima del límite amelocementario hasta encontrar el corte vertical, con disco metálico (Ref: 911 H 104180). Las longitudes fueron tomadas con un dentímetro (Maillefer, Ballaigues, Suiza). Este procedimiento se realizó con motor (NSK ultímate 500 con 30 revoluciones) refrigerado con aire y agua.

Se determinó el eje axial del diente. Se ubicó la punta paralelizadora del paralelómetro y el diente se posicionó con cera adherido a la punta paralelizadora coincidiendo con el eje axial determinado. Los dientes se sumergieron hasta antes del límite amelocementario $2 \mathrm{~mm}$ simulando el límite de la cresta alveolar y luego se llevaron a bloques de acrílico con medidas de $17 \times 20 \mathrm{~mm}$ en estado plástico hasta su polimerización por seis segundos. Luego la porción coronal fue sumergida en un cubo de las mismas dimensiones y la unión de estos fue en junta a tope. Las paredes del cubo acrílico (superior e inferior) guardó paralelismo y perpendicularidad con relación a los platos de la máquina Shimatzu.

Todos los grupos llevaron en su porción coronal cofias metálicas calibradas a $0,5 \mathrm{~mm}$, elaboradas en el sistema adapta de la casa Vego y selladas con cera de sellado de la casa Degudent, enceradas, posteriormente revestidas en un anillo con su respectivo bebedero y se revistió con revesti- miento de grano fino (cerafina) y se sometieron al proceso de evaporación de cera y coladas con metal-base (remaniun), se retiraron del anillo y se inició el proceso de recuperación y pulido y colado. Las cofias fueron cementadas con cemento Calibra (Dentsply).

Los tres grupos fueron sometidos a fuerzas compresivas en la máquina universal de ensayos (Shimadzu Autograph AG-I 250). Con el objetivo de aplicar la fuerza que normalmente soportan los premolares superiores que oscila entre 334 Nws. Los parámetros a tener en cuenta corresponden a la duración del ciclo de masticación que es 0.08 segundos sobre el eje axial del diente. En la maquina se utilizó 0.05 segundos.

La información obtenida se registró en curva de esfuerzo/deformación. Posteriormente los cubos fueron extraídos y examinados clínica y radiográficamente para confirmar la presencia o no de una fractura y el nivel en que estaba ubicadada. Los datos fueron registrados en el formato de recolección de información y sometidos a la prueba estadística indicada.

\section{Análisis estadístico}

En el análisis univariado se presentaron frecuencias y porcentajes con las variables nominales de acuerdo con su distribución, las numéricas se presentaron por medio de medidas de tendencia central como la media y de dispersión como la desviación estándar, el mínimo y máximo.

En el análisis bivariado se aplicaron la prueba estadística Chi cuadrado, t de Student y Anova, previa prueba de normalidad (Shapiro-Wilk), con el fin de realizar inferencias entre las variables de estudio. Se consideraron significativos los estadísticos con un valor de $\mathrm{p}<0,05$.

\section{Consideraciones éticas}

El presente estudio no representó ningún tipo de riesgo, según la resolución 08430 de 1993 del Ministerio de Salud de Colombia en la cual se describen los procedimientos a realizar en investigaciones en salud y se clasifican según el nivel de riesgo.

\section{RESULTADOS}

Del total de dientes evaluados en el grupo de poste colado, el $29 \%$ presentó fractura, la mitad de ellos a nivel medio y los restantes a nivel coronal y apical, 25\% respectivamente. Por su parte en el grupo de dientes restaurados con complementador solo se evidenció fractura en un premolar (7\%) a nivel medio (Tabla 1 ). 
Tabla 1. Asociación entre la técnica de complementación y las variables de resultado.

\begin{tabular}{|c|c|c|c|c|c|c|c|}
\hline \multirow{3}{*}{ Variable } & \multicolumn{6}{|c|}{ Técnica de complementación } & \multirow{3}{*}{ Valor $\mathrm{p}$} \\
\hline & \multicolumn{2}{|c|}{ Poste colado } & \multicolumn{2}{|c|}{ Poste fibra de vidrio } & \multicolumn{2}{|c|}{ Complementador } & \\
\hline & $\mathrm{N}^{\mathrm{o}}$ & $\%$ & $\mathrm{~N}^{\circ}$ & $\%$ & $\mathrm{~N}^{\circ}$ & $\%$ & \\
\hline Resistencia a la fractura & & & & & & & 0,041 \\
\hline $\mathrm{Si}$ & 10 & 71,43 & 15 & 100 & 14 & 93,33 & \\
\hline No & 4 & 28,57 & 0 & 0 & 1 & 6,67 & \\
\hline Nivel fractura & & & & & & & 0,659 \\
\hline Coronal & 1 & 25,00 & 0 & 0 & 0 & 0 & \\
\hline Medio & 2 & 50,00 & 0 & 0 & 1 & 100 & \\
\hline Apical & 1 & 25,00 & 0 & 0 & 0 & 0 & \\
\hline
\end{tabular}

"Chi cuadrado.

De acuerdo con los resultados del estadístico de Shapiro-Wilk, la variable numérica fuerza, tuvo un comportamiento normal ( $p>0,05)$, por tal razón se escogieron para el análisis inferencial las pruebas de Chi cuadrado, t de Student y Anova.

Se observó significancia estadística entre la técnica de complementación y la resistencia a la fractura $(p=0,041)$, lo que demuestra que no existe independencia entre la técnica y la fractura dental de dientes tratados endodonticamente. Caso contrario entre el nivel de fractura y la técnica, dado que el estadístico de $\mathrm{Chi}^{2}$ no fue significativo $(p=0,659)$, es decir, estas variables son independientes (Tabla 1).

En general, para los dientes que no resistieron a la fractura el mínimo de fuerza aplicada hasta el momento de la falla fue de 2.195 y el máximo de 6.331 (Figura 1) y con un $95 \%$ de confianza se asume que el valor verdadero de la media esta entre 3.592 y $5.003 \mathrm{Nw}$ (Me. 4.298 De. 1.323,5) (Tabla 2).

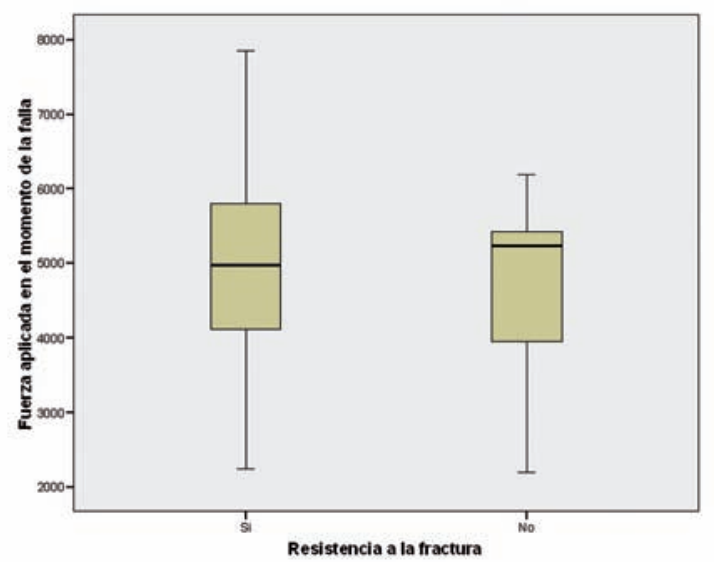

Figura 1. Resistencia a la fractura versus fuerza aplicada en el momento de la falla.

Además, la tabla 2 muestra la distribución de los promedios de la fuerza aplicada en el momento de la falla con relación al nivel de fractura, observándose mayor cantidad de fuerza para la falla en las fracturas ocurridas a nivel medio de los premolares, en promedio 4.939 Nw (De. 1.052,42 IC 95\% 3.261 - 6.616).

Tabla 2. Distribución de las medias de la fuerza aplicada en el momento de la falla con relación a las variables de resultado.

\begin{tabular}{lcccc}
\hline \multicolumn{1}{c}{ Variable } & Me & De & IC $95 \%$ & Valor $\mathbf{p}$ \\
\hline Resistencia a la fractura & & & & $0,383^{*}$ \\
$\mathrm{Si}$ & $4.648,12$ & $1.375,40$ & $4.224-5.071$ & \\
No & $4.297,81$ & $1.323,50$ & $3.592-5.003$ & \\
Nivel fractura & & & & $0,382^{* *}$ \\
Coronal & $3.980,36$ & $1.390,21$ & $3.046-4.914$ & \\
Medio & $4.939,00$ & $1.054,42$ & $3.261-6.616$ & \\
Apical & $5.525,00$ & & & \\
\hline
\end{tabular}

*t de Student, ${ }^{* *}$ Anova 
Dado que los valores arrojados en el análisis estadístico no son significativos se asume que las medias de la fuerza son similares tanto para la resistencia como para el nivel de fractura, valor de $\mathrm{p}=0,383 \mathrm{y}$ $\mathrm{p}=0,382$ respectivamente (Tabla 2 ).

En la figura 2 se observa que en promedio, fue mayor la fuerza aplicada en los postes colados para que ocurriera la falla (Me. 4.980 De. 1.389,55) que en las demás técnicas de complementación.

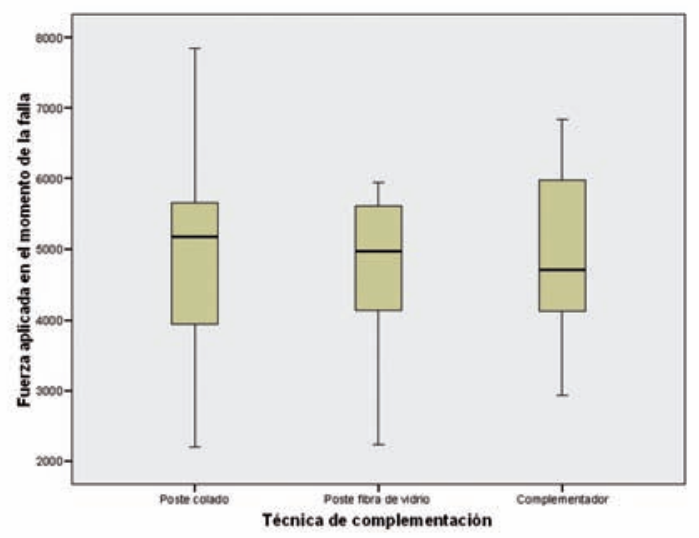

Figura 2. Cantidad de fuerza aplicada en el momento de la falla en cada técnica de complementación.

\section{DISCUSIÓN}

Los tres tipos de rehabilitación seleccionados para este estudio se tuvieron en cuenta debido a que son una forma común de restaurar dientes tratados endodónticamente en las diferentes situaciones clínicas en que es necesario reforzar el remanente coronal o crear un muñón que permita la retención del la futura rehabilitación.

El valor de la carga aplicado se seleccionó después de una revisión de la magnitud de las fuerzas masticatorias cada muestra se inicio con una fuerza de 200 newtons como promedio de la fuerza de mordida aplicada por los dientes premolares, a una carga constante con una velocidad de $0.05 \mathrm{~mm} / \mathrm{min}$ hasta la falla del material, similar a la aplicadas en el estudio de Naranjo y colaboradores en el 2004.,9

En el presente estudio se encontró que los dientes rehabilitados con núcleo colado presentaron menor resistencia a la fractura que los dientes rehabilitados con postes de fibra de vidrio seguida de los complementadores. Sin embargo la mayor fuerza aplicada se dio en los dientes con núcleo colados igual al estudio realizado por Schwartz y Robbins en el 2004. ${ }^{10}$

Lo anterior, permite concluir que los núcleos colados son más rígidos y por esto resisten mas aplicación de fuerza, pero al actuar dentro de una matriz de dentina con un modulo de elasticidad inferior conllevan a la fractura radicular en sitios no favorables o recuperables como la porción apical o media radicular; aspecto que clínicamente llevaría a una perdida dentaria. ${ }^{11}$ Contrario a los postes de fibra vidrio, debido a que el modulo de elasticidad (29 GPA) es similar al de la dentina (18.6 GPA) en comparación con el modulo elástico de los postes colados (90 GpA). ${ }^{8,12}$

Al tener en cuenta que el modulo de elasticidad es la rigidez de un material como lo define Guzman, ${ }^{13}$ y que es un factor influyente en la forma como se comporta un material ante una fuerza, ya que si los módulos elásticos son similares, el comportamiento mecánico será similar y el riesgo de fractura menor.

En este estudio las coronas clínicas se prepararon y se cubrieron con una cofia de metal base calibrada y cementada, sometidas a fuerzas compresivas mediante la maquina Shimatzu partiendo de 200 $\mathrm{Nw}$ y con fuerza incremental cada $0.05 \mathrm{~mm} / \mathrm{min}$ se aplico la fuerza con la maquina universal y se graficó como una curva para cada elemento de la muestra en la que se evidencio la caída de la curva después de un largo ascenso, momento interpretado como una falla en la que se observo una caída del $25 \%$ o mas de la fuerza aplicada esta misma situación se evidencio en el estudio referenciado por Tan y colaboradores. ${ }^{14}$

De esta interpretación de la grafica se asume que los postes colados presentaron la mayor cantidad de resistencia a la fuerzas compresivas llegando a un valor máximo aproximado de $8.000 \mathrm{Nw}$, así también observamos que este grupo soporto la mayor carga en fuerzas y niveles de fractura mas desfavorable debido a que el modulo de elasticidad de los postes colados en oro tipo III es mayor al modulo de la elasticidad de la dentina como lo plantea Wagnild y Mueller en el 2002. ${ }^{3}$

En el grupo de postes de fibra de vidrio se observó que en promedio, la fuerza hasta el momento de la falla fue de aproximadamente $4.900 \mathrm{Nw}$, no siendo evidente la fractura en ninguno de los elementos de la muestra. En este estudio puede concluirse que los postes de fibra de vidrio tienen un modulo elástico que se acerca al de la dentina para reducir la concentración del estrés evitando la tasa de fracaso como lo referencia Akkayam en el $2002 .{ }^{15}$ En el grupo de los complementadores el promedio de la fuerza hasta el momento de la falla fue menor y se evidenció una fractura a nivel de tercio medio la cual es desfavorable, sustentando que no deben ser empleados clínicamente cuando la destrucción coronal es igual o mayor al 50\%. 


\section{Conclusiones}

Los dientes con postes colados resisten mayor fuerza aplicada pero tienen una mayor probabilidad de fractura en un nivel no favorable.

Los dientes con postes en fibra de vidrio resistieron menor fuerza aplicada al momento de la falla y no se evidenció fractura.

Los dientes con complementador presentaron menor resistencia a la fractura y un nivel de fractura no favorable.

\section{BIBLIOGRAFÍA}

1. Healey HJ. Endodontics, St. Louis. En: The C.V. Mosby Company. 1960. p. 267-68.

2. Linde A. Uso de composites en combinación con un poste intrarradicular como muñon de una pieza tratada endodonticamente, aspectos clínicos de la técnica. Quintessence 1995; 8: 10 - 16.

3. Wagnild GW, Mueller KI. Restauración de los dientes tratados endodónticamente. En: Vías de la Pulpa. Madrid: Mosby; 2002. p. 763-95.

4. Salameh Z, Sorrentino R, Papacchini F, Ounsi H, Tashkandi $\mathrm{E}$, Goracci C, et al. Fracture resistance and failure patterns of endodontically treated mandibular molars restored using resin composite with or without translucent glass fiber posts. J Endod 2006; 32: 752 - 755.

5. Heling I, Gorfil C, Slutzky H, Kopolovic K, Zalkind M, Slutzky I. Endodontic failure caused by inadequate restorative procedures: review and treatment recommendations. J Prosthet Dent 2002; 87: 674 - 678.

6. Cleghorn B, Christie W, Dong C. The root and root canal morphology of the human mandibular first premolar: a literature review. J Endod 2007; 33: 509 - 516.

7. Freeman MA, Nicholls JI, Kydd WL, Harrington GW. Leakage associated with load fatigue induced preliminary failure of full crowns placed over three different post and core systems. J Endod 1998; 24: 26 - 32.

8. Naranjo M, Ortiz P, Osorio CL, Sepulveda JR. Comportamiento de dos sistemas de postes prefabricados reconstruidos con resina sometidos a carga cíclica. Estudio piloto. Rev CES Odont 2004; 17: 31 - 38.

9. Naranjo M, Ortiz P, Diaz M, Gomez M, Patiño MC. Resistencia a la fractura de dientes intactos y restaurados con resina sometidos a carga constante. Rev CES Odont 2007; 20: $31-38$.

10. Schwartz RS, Robbins JW. Post placement and restoration of endodontically treated teeth: A literature Review. J Endod 2004; 30: 289 - 301.

11. Christennsen GJ, Gilmore HW, Guyer SE, Leekowitz W, Molone WF. Post and core systems: which one is best. J Prosthet Dent 1982; 48: 27-38.

12. Mohammadi N, Kabnamoii M, Yeganed P, Navimipour E. Effect of fiber post and cusp coverage on fracture resistance of endodontically treated maxillary premolars directly restored with composite resin. J Endod 2009; 10: $1428-1432$.

13. Guzman HJ. Biomateriales Odontológicos de uso clínicos. 4ta. Edición. Bogotá: Ecoe ediciones; 2006.

14. Johnson WT, Dawson D. In vitro fracture resistance of endodontically treated central incisors with varying ferrule heights and configurations. J Prosthet Dent 2005; 93: 331 $-336$.

15. Akkayan B. Resistance to fracture of endodontically treated teeth restored with different post systems. Eur J Prosthodont Restor Dent 2002; 87: 431 - 437.

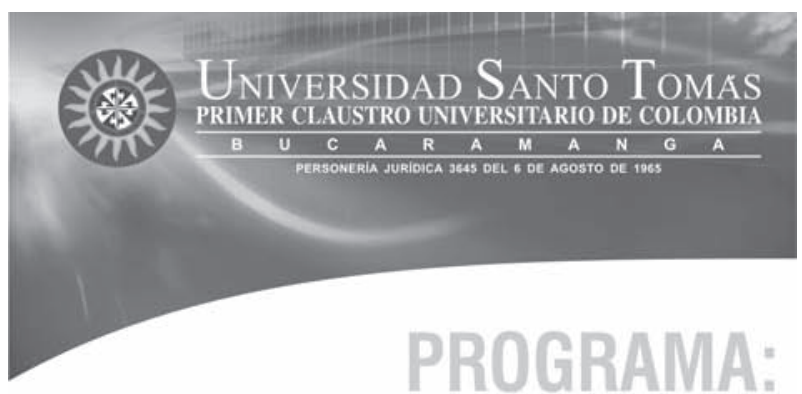

\section{ODONTOLOGÍA}

SNIES 1097

Acreditación de Alta Calidad 517 del 6 de febrero de 2008

\section{www.ustabuca.edu.co}

e-mail: mercadeo@mail.ustabuca.edu.co

6800801 Ext. 1801 - 1263 - 1264 - Linea Gratuita 018000917044

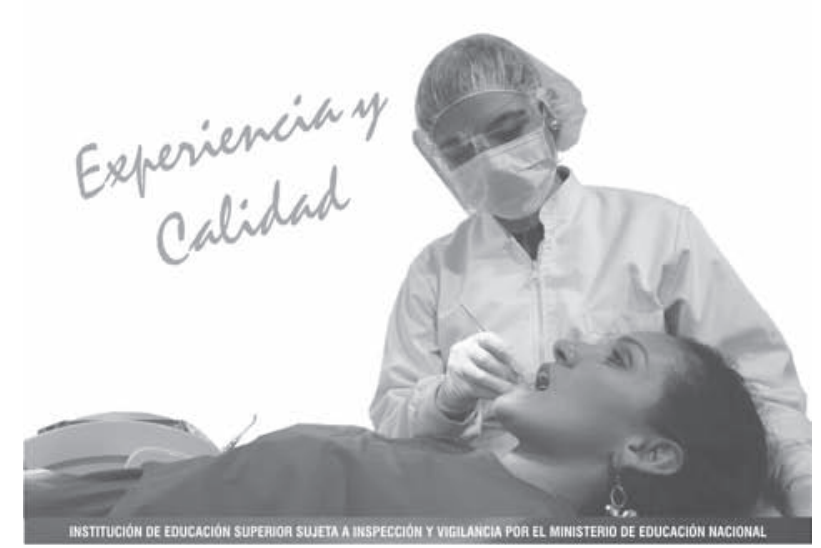

88 